\title{
Influence of Tissue Anisotropy on Molecular Communication
}

\author{
Muneer M. Al-Zu'bi, Ananda S. Mohan, and Steve S.H. Ling
}

Abstract-Many biological tissues inside the human body exhibit highly anisotropic diffusion properties; for example, tissues of the nervous system and white matter in the brain. Here, we present an improved stochastic molecular communication framework to model interaction between bionanomachines in three-dimensional (3D) anisotropic brain micro-environment. The results obtained using stochastic particle-based simulation model are validated with analytical expressions. We also derive expressions for peak amplitude and peak time for the received molecular signal. The results demonstrate that the channel impulse response in anisotropic biological media depends significantly on the diffusion tensor as well as on the locations of the nanomachines.

Keywords-Bionanomachine, channel impulse response, stochastic simulation, molecular communication, anisotropy.

\section{INTRODUCTION}

Molecular communication via diffusion $(\mathrm{MCvD})$ is a promising technique for exchange of biochemical information between either natural biological cells or synthetic bionanomachines via free diffusion of molecules [1]. It can be used in many biomedical applications such as targeted drug $\rightarrow$ delivery, Lab-on-Chip, and heath monitoring systems [2]. Many studies on molecular communication in isotropic biological media (channels) have been investigated in the literature, e.g., [3-6]. The information rate that could be achievable for molecular communication system with passive and active transport of information molecules on a microfluidic chip was derived in [7]. Modelling of a microfluidic molecular communication system with convection, diffusion, and reaction was given in [8].

The isotropic assumption could be sufficient for applications where the media (channel) has identical structure and diffusion properties in all directions. However, the microstructures of several biological tissues (e.g., fibrous tissues) inside the body are highly anisotropic having direction dependent diffusion coefficients. Examples include tissues of the nervous system, such as human spinal cord, sciatic nerve, and tibial nerve, which are highly anisotropic [9]. The fibrous tissues such as brain white matter are also anisotropic because of the parallel orientation of their nerve fiber tracts whereas the grey matter and cerebrospinal fluid (CSF) demonstrate isotropic properties [10]-[11]. It was also shown that the structure and orientation of collagen fibers in extracellular matrix (ECM) of tumors leads to diffusion anisotropy [12]. The mathematical and simulation models provide a practical alternative to estimate molecular interaction and concentration in various anisotropic tissues within the body by incorporating the experimentally determined parameters. Thus, it is important that the anisotropy be included in modeling of molecular communication in biological microenvironments for accurate prediction. Moreover, modelling of the diffusive environment by calculating its channel impulse response (CIR) may help in extending the generalized modelling to more complex biological microenvironments and molecular sources via the mathematical convolution. This assumes greater significance as the development of drug-loaded bio-nano robots becoming a reality. Recently, an autonomous programmed DNA nanorobot has been reported using DNA origami to transport drug payloads and present them specifically at tumours in response to a molecular trigger in vivo [13].

In this paper, we propose a stochastic simulation model for anisotropic channel for a molecular communication via diffusion (MCvD) where a point-like transmitting nanomachine (TN) communicates with a passive receiving nanomachine $(\mathrm{RN})$ placed inside a three-dimensional (3D) anisotropic brain microenvironment. The stochastic particlebased simulation model is then validated using analytically derived channel impulse response (CIR). In this model, the TN may act as a drug-loaded nanomachine while the RN could be a site of action (e.g., diseased cell) inside the brain tissue. Here, the transmitted information can correspond to either sending a trigger signal or releasing a drug payload to the site of action. Moreover, in this work we model brain anisotropic ECM using effective diffusion coefficient which include effect of various tissue properties. Since the simulated time needed for molecules to diffuse between the source and destination is very short, we assume the ECM cells to be stationary (immobile) and will not grow or divide during the simulation time similar to that in [14]. For anisotropic media, the effective diffusion tensor incorporates crucial information which can be represented by an effective diffusion ellipsoid. In this paper, we also obtain the diffusion ellipsoid using the particle simulator which is validated with analytical results.

The paper is organized as follows. In section II, the system model, stochastic particle-based simulation model and analytical expressions for channel impulse response (CIR), the peak amplitude, and the peak time for anisotropy MCvD are presented. The simulation and analytical results are given in section III. Section IV offers brief conclusions based on the work presented in the paper. 


\section{SYSTEM MODEL}

A schematic representation of the molecular communication via diffusion (MCvD) model in an unbounded anisotropic biological micro-environment is shown in Fig. 1. The system consists of a point-like transmitter bio-nanomachine (TN) that emits information molecules which diffuse randomly to reach a receiver bio-nanomachine (or any targeted tissue) in a 3D anisotropic biological microenvironment (channel).

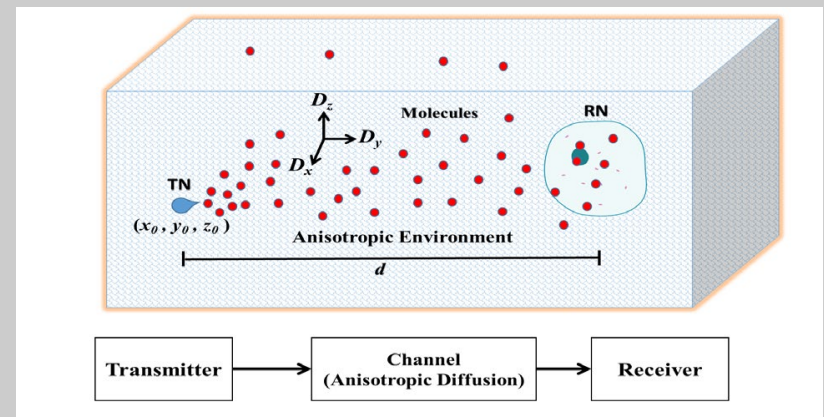

Fig. 1. Molecular communication in anisotropic biological medium.

Here, we assume that the $\mathrm{RN}$ is a virtual spherical observer (i.e., passive receiver) with a volume, $V_{R}$, and radius, $r_{R}$. Due to the tissue anisotropy, the diffusion becomes anisotropic and hence tensor diffusion coefficient must be employed in the modelling. Then, expressions for the channel impulse response (CIR), peak amplitude, and peak time are obtained. Anisotropic diffusion is mathematically described by Fick's second law of diffusion [15],

$$
\frac{\partial C(x, y, z, t)}{\partial t}=\nabla(\mathbf{D} \nabla C(x, y, z, t))
$$

where, $C(x, y, z, t)$ is the concentration distribution, $\nabla$ is the vector differential operator, and $\mathbf{D}$ is the diffusion tensor.

In 3-D anisotropic diffusive media, the diffusion is described by a [3×3] diffusion tensor $(\mathbf{D})$. The diagonal elements of the diffusion tensor represent diffusion coefficients along each coordinate axis. The off-diagonal elements represent the correlation between the random motions in each pair of directions. In this work, we consider the diffusion tensor to have zeros in the off-diagonal elements. This means that the structure and characteristic of the media in the direction of $x$, $y$, and $z$-axes are non-identical.

Using [15, eq. 1.10], Fick's second law of diffusion (1) for 3D anisotropic diffusion tensor can be expressed as

$$
\begin{array}{r}
\frac{\partial C(x, y, z, t)}{\partial t}=D_{x} \frac{\partial^{2} C(x, y, z, t)}{\partial x^{2}}+D_{y} \frac{\partial^{2} C(x, y, z, t)}{\partial y^{2}} \\
+D_{z} \frac{\partial^{2} C(x, y, z, t)}{\partial z^{2}}
\end{array}
$$

where $D_{x}, D_{x}$, and $D_{x}$ are the diffusion coefficients along $x, y$, and $z$ directions, respectively.

An instantaneous emission of the information molecules by the $\mathrm{TN}$ at the position $r_{0}=\left(x_{0}, y_{0}, z_{0}\right)$ at initial time $t=0$ in an unbounded medium is characterized using the following initial and boundary conditions:

$$
\begin{gathered}
f_{0}=\delta\left(x-x_{0}\right) \delta\left(y-y_{0}\right) \delta\left(z-z_{0}\right) \delta(t) \\
\lim _{(x, y, z) \rightarrow \pm \infty} C(x, y, z, t)=0
\end{gathered}
$$

where, $\delta(\cdot)$ is Dirac delta function.

\section{A. Analytical expressions}

The expressions for molecular displacement probability for anisotropic media are available in the literature which have been widely used in diffusion and NMR imaging as well as for the estimation of diffusion tensor [10, 11], [16]. A mathematical expression for molecular distribution (or displacement probability distribution function) due to 3-D anisotropic diffusion is re-derived here [11]. Using method of separation of variables, the solution of the diffusion equation (2) for the conditions (3)-(4) will lead to the Green's function that can be expressed as

$$
C(x, y, z, t)=G_{x}(x, t) \cdot G_{y}(y, t) \cdot G_{z}(z, t)
$$

The Green's function can be expressed as a product of the solutions of the one-dimensional (1-D) diffusion equations, i.e., $G_{u}(u, t)$, along each direction $u \in\{x, y, z\}$. The function $G_{u}(u, t)$ is given as,

$$
G_{u}(u, t)=\frac{1}{\sqrt{4 \pi D_{u} t}} \exp \left(-\left(u-u_{0}\right)^{2} / 4 t D_{u}\right)
$$

Now, the displacement probability distribution function can be obtained by substituting (6) in (5) as

$$
h_{A}(x, y, z, t)=\frac{1}{\sqrt{D_{x} D_{y} D_{z}}(4 \pi t)^{3 / 2}} \exp \left(-\frac{\lambda}{4 t}\right)
$$

where,

$$
\lambda=\frac{\left(x-x_{0}\right)^{2}}{D_{x}}+\frac{\left(y-y_{0}\right)^{2}}{D_{y}}+\frac{\left(z-z_{0}\right)^{2}}{D_{z}}
$$

As a special case, the displacement probability distribution function for 3D isotropic media (e.g., gray matter in brain), can be obtained by making all diffusion coefficients equal to a single diffusion coefficient $D$.

$$
h_{I}(x, y, z, t)=\frac{1}{(4 \pi D t)^{3 / 2}} \exp \left(-\frac{d^{2}}{4 D t}\right)
$$

where, $d$ is the separation distance between the TN and RN.

The peak time instant is the time instant at which the displacement probability distribution has maximum amplitude and it is obtained as

$$
t_{\max }=\lambda / 6
$$

Now, substituting (10) in (7), we get the peak amplitude of the displacement probability distribution.

$$
h_{\max }=3^{3 / 2} /\left[\sqrt{D_{x} D_{y} D_{z}}(2 \lambda \pi e)^{3 / 2}\right]
$$


The molecular concentration inside the passive receiver volume is assumed to be uniformly distributed. Thus, the expected number of the received molecules (or the CIR) by the $\mathrm{RN}$ can be expressed as

$$
N=Q V_{R} h
$$

where, $Q$ is the total released molecules at time $t=0$ and $h$ is given by (7) or (9).

\section{B. Stochastic particle-based simulation model}

We present a particle-based simulator for molecular communication between point-like TN and spherical passive (i.e., virtual observer) inside 3D anisotropic microenvironment that has different diffusion properties along each axis. The simulated results are compared with the analytical results for validation. The simulation is developed using MATLAB software package. In this stochastic simulation model, the simulation time $T$ is divided into $N$ small time steps $\Delta t$. The number of the information molecules and their accurate locations are tracked and recorded at the end of each time step. Firstly, the information molecules are instantaneously emitted from a point $\mathrm{TN}$ that is located at the position $r_{0}=\left(x_{0}, y_{0}, z_{0}\right)$. Then, they are made to move randomly following Brownian motion in all the directions. Since the simulation environment is anisotropic, the randomly propagating molecules experience different diffusivities in each direction and consequently result in different displacements along each axis. The precise location of each molecule is tracked and updated at each time step as follows:

$$
\left(x_{i}, y_{i}, z_{i}\right)=\left(x_{i-1}, y_{i-1}, z_{i-1}\right)+\left(\Delta x_{i}, \Delta y_{i}, \Delta z_{i}\right)
$$

where, $i=1,2, \ldots, \mathrm{N}, \mathrm{N}$ is the total number of the simulation time steps, the coordinate $\left(x_{i}, y_{i}, z_{i}\right)$ is the current molecule position at the $i^{\text {th }}$ time step, the coordinate $\left(x_{i-1}, y_{i-1}, z_{i-1}\right)$ is the previous molecule position at the $(i-1)^{t h}$ time step, and $\left(\Delta x_{i}, \Delta y_{i}, \Delta z_{i}\right)$ is the random displacements over each spatial axis at $i^{\text {th }}$ time step which follows the normal distribution $N\left(0, \sigma_{u}^{2}\right)$ with zero-mean and different variances, i.e., $\sigma_{u}^{2}=2 D_{u} \Delta t$, for $u \in\{x, y, z\}$. Finally, at the end of each time step, the total number of the information molecules that reach the passive receiver is recorded which is denoted as the received signal or the CIR.

\section{RESULTS}

In this section, the analytical and simulation results on channel impulse response (CIR) and its peak amplitude and peak time are plotted for different diffusion tensors and different locations and distances between the nanomachines. For the sake of analysis, we use the following simulation parameters: number of released molecules is equal to $10^{5}$ molecules, time step is equal to $10^{-4} \mathrm{~s}, \mathrm{RN}$ radius is equal to $2 \mu \mathrm{m}$, TN is located at the origin, and number of iterations is equal to 100 iterations. In this model, the extracellular space (ECS) is an anisotropic brain region that contain many aligned fibres along which the axial diffusion is faster than the diffusion across the radial directions. For sake of analysis and based on available data [16], the diffusion tensor of Alexa
Fluor 488 (AF) molecules in anisotropic ECS is chosen to compute the CIR as shown in Fig. 2 where the results are plotted for different $\mathrm{RN}$ locations. To get a fair comparison, the $\mathrm{RN}$ is moved between various locations but with identical distance from the TN (the origin point), i.e., $d=10 \mu \mathrm{m}$. As shown in Fig. 2, the CIR shows higher amplitude (and lower peak time) when the $\mathrm{RN}$ is located at the position $(10,0,0) \mu m$ on the $\mathrm{x}$-axis but as the RN moves away from the $\mathrm{x}$-axis, e.g., when the $\mathrm{RN}$ is located at $(5.7,5.7,5.7) \mu m$, the amplitude decreases with corresponding increase in the peak time. The worst case with lowest amplitude and longest peak time happens when the $\mathrm{RN}$ is located on $y$ - or $z$-axes. This may be due to fact that the diffusion tensor exhibits diffusion preference towards $\mathrm{x}$-axis direction rather than $\mathrm{y}$ - and $\mathrm{z}$ - axes. Thus, if the $\mathrm{RN}$ is located near the $\mathrm{x}$-axis, it will fall within the diffusion ellipsoid of the molecule distribution and thus a higher number of molecules may reach the $\mathrm{RN}$ within a shorter time.

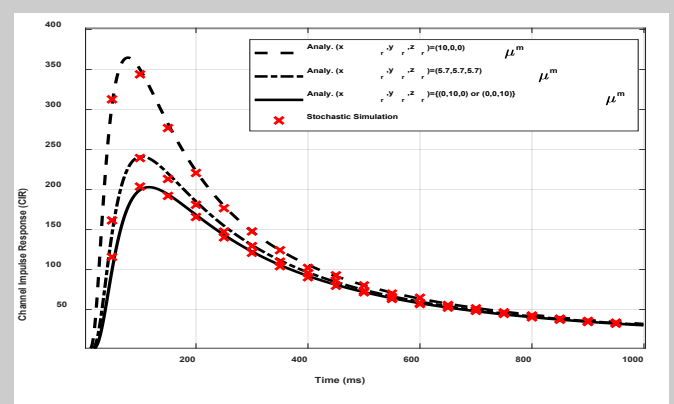

Fig. 2. Channel impulse response (CIR) for various locations of the RN in 3D anisotropic extracellular space for $D=(2.1,1.4,1.4) \times 10^{-10} \mathrm{~m}^{2} / \mathrm{s}$.

The anisotropic diffusion tensor can be viewed using the diffusion ellipsoid whose main axis is parallel to the principal diffusion direction whereas for isotropic diffusion, the ellipsoid becomes a sphere [11]. Fig.3 shows visualization of the diffusion ellipsoid for the diffusive molecules in $3 \mathrm{D}$ anisotropic environment with different diffusion tensors obtained from our particle simulator. The molecular distributions (diffusion ellipsoids) are captured from the simulator at a time instant equal to the peak time of the CIR when the $\mathrm{RN}$ is located at the point $(10,0,0) \mu \mathrm{m}$. The darker regions represent higher molecules density and the larger spread area represents the longer diffusion time. As shown in Fig. 3a, the diffusion is identical in an isotropic environment when $D=(5,5,5) \times 10^{-9} \mathrm{~m}^{2} / \mathrm{s}$, and the diffusion ellipsoid is a sphere. Thus, for isotropic case, whatever be the RN location (as long as it is equidistant from TN), the CIR will have identical characteristics. However, as shown in Fig. $3 \mathrm{~b}$ for an anisotropic environment with $D=(5,1,1) \times 10^{-9} \mathrm{~m}^{2} / \mathrm{s}$, the diffusion ellipsoid shows preference in the direction of $\mathrm{x}$-axis (major axis) while the diffusion is restricted but identical in other directions (minor axes). One can observe circular diffusion spread in $y-z$ plane because the diffusion coefficient components are equal. Therefore, the highest amplitude and lowest peak time can be achieved when the $\mathrm{RN}$ is positioned on $\mathrm{x}$-axis, i.e., the major axis of the diffusion ellipsoid. 
The peak amplitude and peak time of the CIR as a function of the separation distance between TN and RN are plotted in Fig. 4, for various diffusion tensors. As expected, the peak amplitude shows increasing trend while the peak time shows decreasing trend as the TN-RN separation distance increases. As the separation distance increases lesser number of molecules may succeed in reaching the $\mathrm{RN}$ and thus only few numbers of molecules will be detected by the RN. Moreover, the molecules will take longer time to reach the $\mathrm{RN}$ as the separation distance increases.
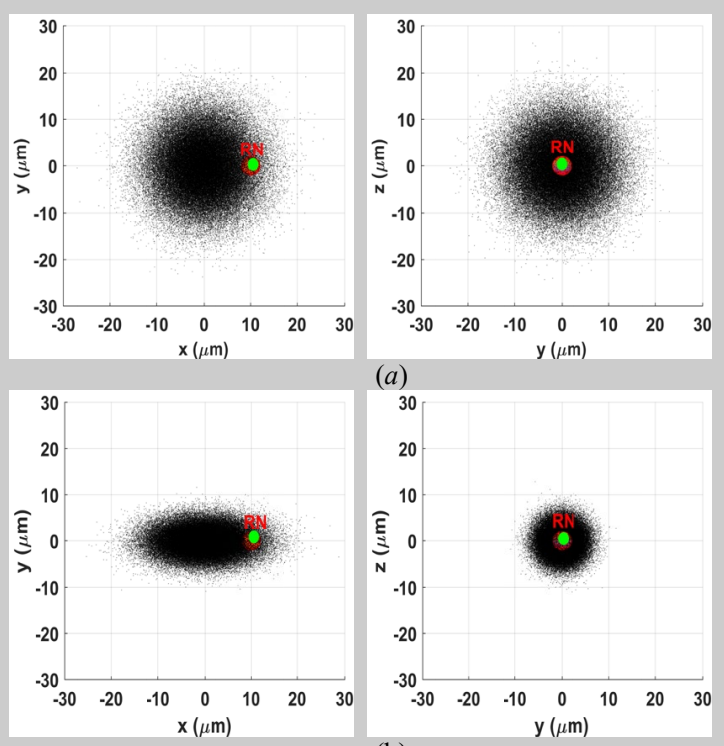

(b)

$54 \mathrm{pt}$ 0.75 in $19.1 \mathrm{~mm}$
Fig. 3. Visualization of diffusion ellipsoid in 3D anisotropic environment for a. $D=(5,5,5) \times 10^{-9} \mathrm{~m}^{2} / \mathrm{s}$ and b. $D=(5,1,1) \times 10^{-9} \mathrm{~m}^{2} / \mathrm{s}$.

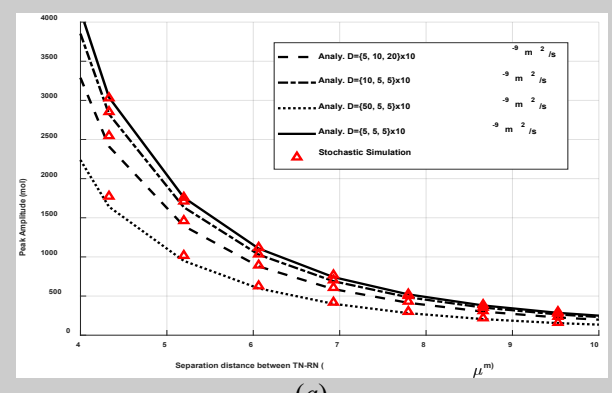

(a)

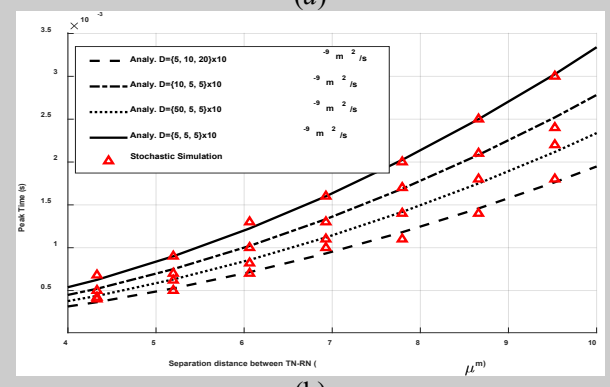

(b)

Fig. 4. Peak amplitude and peak time as function of TN-RN separation distance for various diffusion tensors.

\section{CONCLUSION}

In this paper, we present the impact of tissue anisotropy on molecular communication by comparing the simulated and analytical channel impulse response (CIR) in an anisotropic diffusive biological microenvironment. We present a stochastic anisotropic particle-based simulator which has been validated using analytical results. We derive expressions for the peak amplitude and peak time of the CIR. The results show that the peak amplitude of the CIR for anisotropic environment highly depends on the diffusion tensor unlike the isotropic case where the peak amplitude is independent of diffusion coefficient. Moreover, the amplitude and time characteristics of the channel impulse response not only depend on the distance between the nanomachines as in the case of isotropic diffusion, but they also show high dependency on the locations of the nanomachines inside 3-D environments. Thus, the results of this work could help in the design of drug delivery systems and bio-nanomachines as well as in the applications of In-Body nano networks.

\section{REFERENCES}

[1] P.-C. Yeh et al., "A new frontier of wireless communication theory: diffusion-based molecular communications," IEEE Wireless Communications, vol. 19, no. 5, 2012.

[2] T. Nakano, M. J. Moore, F. Wei, A. V. Vasilakos, and J. Shuai, "Molecular communication and networking: Opportunities and challenges," IEEE transactions on nanobioscience, vol. 11, no. 2, pp. 135-148, 2012.

[3] M. Pierobon and I. F. Akyildiz, "A physical end-to-end model for molecular communication in nanonetworks," IEEE Journal on Selected Areas in Communications, vol. 28, no. 4, 2010.

[4] D. Kilinc and O. B. Akan, "Receiver design for molecular communication," IEEE Journal on Selected Areas in Communications, vol. 31, no. 12, pp. 705-714, 2013.

[5] A. Noel, K. C. Cheung, and R. Schober, "Optimal receiver design for diffusive molecular communication with flow and additive noise," IEEE transactions on nanobioscience, vol. 13 , no. 3, pp. 350-362, 2014.

[6] A. Noel, K. C. Cheung, and R. Schober, "Improving receiver performance of diffusive molecular communication with enzymes," IEEE Transactions on NanoBioscience, vol. 13, no. 1, pp. 31-43, 2014

[7] N. Farsad, A. W. Eckford, S. Hiyama, and Y. Moritani, "On-chip molecular communication: Analysis and design," IEEE Transactions on NanoBioscience, vol. 11, no. 3, pp. 304-314, 2012.

[8] M. Kuscu and O. B. Akan, "Modeling convection-diffusion-reaction systems for microfluidic molecular communications with surfacebased receivers in Internet of Bio-Nano Things," PloS one, vol. 13, no. 2, p. e0192202, 2018.

[9] C. Beaulieu, "The basis of anisotropic water diffusion in the nervous system-a technical review," NMR in Biomedicine, vol. 15, no. 7-8, pp 435-455, 2002.

[10] P. Mukherjee, J. Berman, S. Chung, C. Hess, and R. Henry, "Diffusion tensor MR imaging and fiber tractography: theoretic underpinnings," American journal of neuroradiology, vol. 29, no. 4, pp. 632-641, 2008.

[11] A. L. Alexander, J. E. Lee, M. Lazar, and A. S. Field, "Diffusion tensor imaging of the brain," Neurotherapeutics, vol. 4, no. 3, pp. 316-329, 2007.

[12] T. Stylianopoulos, B. Diop-Frimpong, L. L. Munn, and R. K. Jain, "Diffusion anisotropy in collagen gels and tumors: the effect of fiber network orientation," Biophysical journal, vol. 99, no. 10, pp. 3119 3128, 2010.

[13] S. Li et al., "A DNA nanorobot functions as a cancer therapeutic in response to a molecular trigger in vivo," vol. 36, no. 3, p. 258, 2018.

[14] K. A. Rejniak, V. Estrella, T. Chen, A. S. Cohen, M. Lloyd, and D. L. J. F. i. o. Morse, "The role of tumor tissue architecture in treatment penetration and efficacy: an integrative study," vol. 3, p. 111, 2013.

[15] J. Crank, The mathematics of diffusion. Oxford university press, 1979

[16] F. Xiao, C. Nicholson, J. Hrabe, and S. Hrabětová, "Diffusion of flexible random-coil dextran polymers measured in anisotropic brain extracellular space by integrative optical imaging," Biophysical journal, vol. 95, no. 3, pp. 1382-1392, 2008. 